\title{
Incidencia del uso de un manual de funciones en las micro y pequeñas empresas orenses
}

\section{Incidence of use of functions manual in micro and small enterprises from el oro}

\author{
Dustin Coronel Cruz \\ David Cartuche Paqui \\ Luis Rodríguez Jiménez \\ Juan Saca Lucero \\ Universidad Técnica de Machala, Ecuador
}

Autor para correspondencia: dustincoronel@hotmail.com; dcartuche@utmachala.edu.ec; lerodriguezj@utmachala.edu.ec; Juan.s2593@gmail.com

Fecha de recepción: 17 de agosto de 2018 Fecha de aceptación: 15 noviembre del 2018

\section{Resumen}

El presente artículo tiene como propósito contrastar el impacto que tiene un manual de funciones documentado y su importancia en las micro y pequeñas empresas de El Oro. El estudio se desarrolló en dos fases, la primera en realizar un estudio documental para fundamental teoría básica de esta herramienta de gestión empresarial, su utilidad y como afecta directamente a las empresas indistintamente cual sea su tamaño como empresa, en la segunda fase se aplicó una investigación de campo, recopilando información con encuestas, las cuales fueron aplicadas en la provincia de El Oro. Los resultados obtenidos indican que existen conflictos notorios durante las jornadas de trabajo, tales como la aglomeración de actividades y confusión por parte de los empleados.

Palabras claves: manual de funciones; herramienta de gestión; eficiencia; micro y pequeñas empresas

\begin{abstract}
The purpose of this paper is to compare the impact of a documented function manual and the importance in the micro and small enterprises of El Oro. The study was carried out in two phases, the first to carry out a documentary study for the basic theory of this in the second phase, a field investigation was applied, collecting information with surveys, which were applied in the province of El Oro. Results obtained indicate that there are notorious conflicts during working days, agglomeration of activities and confusion on the part of the employees.
\end{abstract}

Key words: function's manual; management tool; efficiency; micro and small business 


\section{Introducción}

La problemática constante de las directrices empresariales actuales se ha centrado simplemente en comprender que para alcanzar metas se requiere de un diseño estratégico plenamente definido. Para que lo anteriormente expuesto se efectué, las organizaciones deben realizar componentes de acción para obtener resultados positivos. Para las micro y pequeñas empresas que se enfrentan constantemente a estos contextos, deben poner en marcha la práctica de formalización de actividades, control y su planificación en cada una de sus líneas de trabajo generando un mejoramiento en el ambiente laboral y actividades diarias.

Cada organización que reflexiona hacia el éxito debe tener definida una estructura organizacional para que sea de conocimiento general el grado de responsabilidad del funcionario "Cualquier estructura de acción colectiva o sistema de acción concreta se constituye como un sistema de poder apoyado sobre las incertidumbres inherentes a los problemas a resolver" (Bernal Domínguez \& Herrera Caballero, 2014, p.6). Su determinación jerárquica permite a los colaboradores conocer a que autoridad deben responder y que actividades deberán desempeñar, teniendo en cuenta que un manual de funciones facilita la información.

Para optimizar el funcionamiento organizacional y las prestaciones de servicios mediante un manual de funciones es necesario definir un sistema de información acorde a la estructura organizacional. Según Loyo de Falcón (2015) "Depende de los siguientes factores: seguimiento del rendimiento, utilización de la retroinformación, respuesta del receptor, empatía (colocarse en el lugar del otro), repetición, confianza mutua, eficacia en el momento que se produce la comunicación simplificación y escuchar eficazmente" (p.12). Su implementación incita a la optimización de tiempo a través de una estructura flexible y adaptable basado en una comunicación fluida y precisa.

Del conocimiento que obtenga cada colaborador de sus funciones proviene del proceso de comunicación que desarrolla la organización. Como se cita en Castañeda (2015), "en una organización cada trabajador aprende a través de diferentes mecanismos, así como también lo hacen los grupos de trabajo. A la vez, se puede afirmar que hay aprendizaje en el nivel organizacional cuando el conocimiento se institucionaliza" (p.3). Para este efecto colateral la institución debe utilizar mecanismos de información que personalicen el trabajo de manera concreta y acertada, ya que de la generación de conocimiento que provea la empresa el colaborador estará en condiciones óptimas para prestar su servicio.

La inserción de talento humano a una organización se atribuye un proceso de admisión que tiene que ver con la instrucción del personal sobre las actividades que va a realizar. Castañeda \& Fernández R (2007) afirma que "El aprendizaje organizacional es un proceso de adquisición y transferencia de conocimiento que se da en tres niveles: individual, grupal y organizacional" (p.3). Una de las herramientas que se suelen utilizar es el manual de funciones que familiariza al postulante con sus responsabilidades. funciones es:

Según Gonzales (como se citó en Sotomayor Cun, 2016) establece que un manual de 
Considerado como una herramienta de trabajo en el que se expone de forma clara y precisa el conjunto de normas y tareas que deben cumplir cada una de la persona de la organización, por tanto, éste debe ser elaborado técnicamente, siguiendo los respectivos procedimientos para determinar las rutinas o labores cotidianas de los trabajadores (pág. 30)

El crecimiento progresivo de empresas en la región da apertura a la necesidad de implementar estas herramientas en todos los departamentos, evitando futuras problemáticas, potenciando el control administrativo, finiquitando metas establecidas y mejorando constantemente los procesos de las organizaciones mediante una evaluación de actividades diarias.

Conjuntamente esta investigación busca servir de referente a la comunidad empresarial en cuanto a la importancia de contar con un manual de funciones dentro de las micro y pequeñas empresas, tomando en cuenta que un manual de funciones es aquel documento que contiene, tanto el análisis de diferentes cargos, así como la descripción de los mismos, siendo útil para alcanzar la eficiencia administrativa y facilitar la coordinación de los cargos (Delgado, 2014).

Según lo establece la Comunidad Andina de Naciones (2009), para determinar el tamaño de las empresas, se lo realiza en relación al número de trabajadores que posee y al nivel de ventas anuales, siendo microempresas aquellas que contengan de 1 a 9 empleados y reciban ingresos anuales por ventas de hasta cien mil dólares; a su vez las pequeñas empresas son aquellas que cuenten una cantidad de empleados de 10 a 49 y que reciban ingresos anuales por venta mayores a cien mil y hasta un millón de dólares.

La investigación se encuentra enfocada a determinar la medida en que las micro y pequeñas empresas de la provincia de El Oro resultan afectadas al no contar un manual de funciones. Considerando que en los tiempos actuales un manual de funciones constituye una de las herramientas indispensables para que una empresa sea competitiva y realice de manera eficiente y eficaz sus procesos, alcanzando resultados ideales (León, 2013). Por lo tanto, resulta necesario identificar aquellas razones por las cuáles ciertas empresas no aplican uno actualmente y a su vez conocer el desempeño de aquellas que sí lo aplican, logrando de esta manera determinar la diferencia entre ambos ambientes laborales.

Es decir, la investigación analiza la carencia de un manual de funciones en las micro y pequeñas empresas orenses que afecta el rendimiento laboral de su personal, lo cual resulta en un ambiente donde las funciones y responsabilidades laborales no especificadas conllevan a la duplicación de funciones e inadecuada distribución de actividades, así como a la incompetencia laboral al no contar con perfiles de cargos definidos.

El $5 \%$ de las empresas existentes en la provincia surgen por la motivación de ser independientes y la oportunidad percibida para obtener más que una posición asalariada, fueron las dos razones esenciales para incursionar en una micro y pequeña empresa en lugar de laborar bajo dependencia de un sueldo. Sin embargo, este tipo de empresas en ocasiones se enfrentan a escenarios donde la gerencia no cuenta con los conocimientos dirigénciales y administrativos competentes para mantener una estabilidad eficaz en las operaciones realizadas en todos sus mandos, como la posibilidad de la ausencia de un manual de funciones, creando constantemente 
conflictos en las operaciones empresariales (Magill J, 2004). Estadísticamente datos sobre las microempresas únicamente se obtenían por la agencia de los Estados Unidos para el Desarrollo Internacional (USAID), donde expresa la informalidad de las organizaciones la cual fundamenta la investigación.

La carencia de un manual de funciones da origen a desconciertos laborales, conflictos organizacionales, poca captación de responsabilidades de cargos, bajos estándares de formación e información al personal trabajador en micro y pequeñas empresas. Esta hipótesis es referente a comprobación.

La veracidad de la investigación presente permite obtener datos relevantes del momento actual que conviven las organizaciones micro y pequeñas de la provincia de El Oro en relación al ambiente laboral, por ende, contrastar cuanto influye para mandos dirigénciales con sus empleados el uso de reglamentos internos para descripción de actividades, código ético y de conducta es vital para cada encargado de la administración de sus negocios relevando la importancia de mantener estos manuales de funciones de manera documentada:

\section{Materiales y Métodos}

Para poder abordar el tema de investigación se aplicó este modelo de estudio denominado estudios exploratorios permitiendo detallar a cabalidad aspectos intrínsecos de la materia y la realidad de la empresa, los estudios exploratorios han servido para poder identificar las variables establecida para el desarrollo de la investigación. Cauas (2015) nos da su explicación mencionando que "El objetivo es examinar un tema o problema de investigación poco estudiado o que no ha sido abordado antes. Estos estudios sirven para aumentar el grado de familiaridad con fenómenos relativamente desconocidos" (p.5). Por ello se ha considerado a los estudios exploratorios punto de inicio en toda investigación.

Cauas (2015) menciona que este tipo de estudio permite detallar a cabalidad aspectos intrínsecos de la materia y la realidad de la empresa, los estudios exploratorios han servido para poder identificar las variables establecida para el desarrollo de la investigación. Los diferentes niveles de investigación difieren en el tipo de pregunta que pueden formular" (p.6). Para el éxito de esta metodología de estudio fue necesario abarcar técnicas de recolección de datos que permitan obtener información veraz del estudio realizado, como datos de páginas gubernamentales.

Este tipo de estudio logró identificar características esenciales del presente de la organización. No obstante, en las investigaciones exploratorias no se trazan interrogantes que indiquen la raíz del problema, sino que se examinan de manera superficial, en este nivel de estudio las preguntas están dirigidas mediante taxonomías y esquemas descriptivos; todas las interrogantes están dirigidas hacia las variables (Cauas, 2015).

Los estudios exploratorios también llamados formulativos buscan indagar en el caso de estudio cuyo no haya sido analizado con anticipación y requiera de atención; contribuye atender la reacción de las variables ante una situación determinada (Abreu, 2012). 
Para una mayor profundidad de la investigación se realizó una encuesta como técnica de recolección de datos, cada pregunta se revisó de forma analítica e independiente, de tal manera que logra realizar un juicio de valor que solucione la problemática planteada (Ibáñez \& Egoscozábal, 2008). El eje principal de la investigación es determinar la influencia de ejecutar un manual de funciones dentro de las micro y pequeñas empresas orenses y factores inmersos en las actividades cotidianas de las jornadas diarias con respecto a la variable ambiente laboral.

Para la obtención de los datos, se procedió a encuestar a las empresas de la provincia de El Oro, cuyo universo cuenta con aproximadamente el 4,99\% de las empresas del estado ecuatoriano, es decir, aproximadamente 42.063. Estos datos fueron obtenidos del directorio de empresas del INEC (2015), en el cual se expresa la cantidad de empresas existentes en el país y en cada una de las provincias, así como, el número de empresas existentes de acuerdo a su tamaño y sector económico. Por lo tanto, del total de empresas existentes en la provincia, para la investigación sólo se tomaron en consideración a las micro y pequeñas empresas, las cuales suman un total de 41.217, siendo esta la población de estudio.

\section{Tabla 1. Número de micro y pequeñas empresas}

\begin{tabular}{lllll}
\hline & Ecuador & El Oro & Ecuador & El Oro \\
\hline Total & $100 \%$ & $4,99 \%$ & 842.936 & 42.063 \\
Micro & $90,64 \%$ & $4,52 \%$ & 764.037 & 38.125 \\
Pequeñas & $7,35 \%$ & $0,37 \%$ & 61.956 & 3.092 \\
\hline
\end{tabular}

Fuente. Directorio de empresas 2015 del INEC.

De acuerdo a la Tabla 1, se tiene el total de empresas existentes en el país, tanto en valores nominales como porcentuales, donde se puede observar que el universo de la investigación se encuentra conformado por 38.125 microempresas y tan solo 3.092 pequeñas empresas. Para la determinación de la muestra, según se aprecia en la Tabla 2, se trabajó con un porcentaje de error del 5\% y un intervalo de confianza del 95\%, y una vez aplicada la fórmula de tamaño muestral (Ver Ecuación 1) para una población finita, se determinó que la muestra de la población a encuestar era de 381 empresas.

$$
n=\frac{N Z^{2} p q}{N e^{2}+Z^{2} p q} \quad \text { (1) Formulación de tamaño muestral }
$$

La investigación fue fundamentada a través de la utilización de herramientas metodológicas que agilizan la recolección de datos, para posteriormente sustentar la investigación del cual fueron objeto, ya que esta incluye aspectos tanto sociales como económicos.

Tabla 2. Determinación de la muestra

\begin{tabular}{ll}
\hline Elemento & Valor \\
\hline N (Población) & 41.217 \\
Z(Nivel de confianza) 95\% & 1,96 \\
Z2 & 3,8416 \\
p (Probabilidad de éxito) & 0,5 \\
q (Probabilidad de fracaso) & 0,5
\end{tabular}




\begin{tabular}{ll} 
e (Margen de error) & 0,05 \\
$\mathrm{n}$ (Tamaño de la muestra) & 381 \\
\hline
\end{tabular}

\section{Métodos de nivel teóricos utilizados}

\section{Cuantitativa}

El método cuantitativo es de criterio directo y ecuánime, basándose en la recolección de información variada y dependiente de la población a estudiar. Según el criterio de Arias Valencia (2013) establece que "la investigación cuantitativa se basa en grandes muestras representativas de la población seleccionada, (...). La adecuación de la muestra se determina estadísticamente, igual que su representatividad del total de la población" (p.23).

El efecto del estudio cuantitativo contribuyo con información relevante y característica del momento actual que vive la población estudiada, permitiendo la apertura completa al proceso de derivación inferencial de el por qué la presentación de su realidad (Pita Fernández \& Pértegas Díaz, 2002).

\section{Tipo de investigación aplicada}

\section{Investigación de campo}

La investigación de campo es la recopilación de datos del estudio a realizar. De tal manera, el levantamiento de información tal como las comprobaciones de hechos, los análisis y ejecución de la metodología establecida, los cuales incurren directamente en el lugar de los hechos. Para autores como Utreras (2015) establece que:

Esta área de la investigación es una de las básicas para determinar la influencia de los medios en los públicos perceptores; el valor de los contenidos y su probable aporte al desarrollo cultural y económico de la sociedad y toda una gama de influencias que pueden determinar el comportamiento que se induce mediante los medios a la sociedad (p.86).

El desarrollo de este tipo de investigación se determinó por datos obtenidos del entorno como en el campo digital que provee el gobierno con fuentes que son de carácter social, es decir accesibles o libre de conocimiento que sirvieron como referencia para para determinar datos cuantitativos (Martínez, 2007)

\section{Investigación documental}

La investigación documental consiste en la compilación de información pertinente del estudio a realizar, es de carácter teórico fundamentado únicamente en el pensamiento y criterio de cada autor. Según el juicio de Paz (2014) menciona que "la investigación documental es la búsqueda de una respuesta específica a partir de la indagación en documentos" (p.12).

Este método de estudio permitió que el investigador profundice cada uno de los temas a tratar, evidenciando la importancia de las temáticas establecidas y su pertinencia en la sociedad, los resultados emitidos fueron de carácter inédito 


\section{Métodos de aplicación a la investigación}

\section{Método encuesta}

Para la aplicación de la encuesta se tomó datos reales de páginas gubernamentales como la población y número de empresas, dentro de la república del Ecuador la entidad encargada de mantener un informe estadístico es el Instituto Nacional de Estadística y Censos "INEC". Para la aplicación de la encuesta se referenciaron varios aspectos que agilitaron la investigación, Cauas (2015) menciona que "En estudios, se recogen datos más o menos limitados, que se refieren a grupos relativamente amplios. Interesan más las variables que describen grupos que a los individuos. Es el tipo de investigación más común" (p.7). La encuesta se fue aplicada a empresas dentro del rango de micro y pequeñas empresas situadas en la provincia de El Oro.

Este tipo de metodología permitió obtener datos importantes que permitan responder de manera cualitativa y cuantitativa a la hipótesis planteada, agilizando la sustentación de la investigación e inmediatamente dejando precedentes para futuras interrogantes, ya que se determina el número de micro y pequeñas empresas a encuestar, específicamente de la provincia de EL Oro, dato esencial que sirvió como referente técnico.

\section{Análisis de datos}

En el proyecto de investigación propuesto realizo un análisis de datos de estadística descriptiva, porque se interpreta los datos por medios de cuadros, gráficos y resúmenes; presentándose de manera real sin alterar los resultados obtenidos. Por lo cual se utilizará el programa Microsoft Excel para procesar la información y analizar los resultados obtenidos.

El estudio que se utilizó fue de todo exploratorio y descriptivo, permitió conocer el estado actual de las organizaciones con relación a la incidencia de la utilización de un manual de funciones.

Luego se prosiguió a realizar el estudio macro de datos que se pueden obtener del INEC que expresa el número de micro y pequeñas empresas que existen a nivel nacional, datos de la población Orense, además de la utilización de fuentes documentales, que posteriormente mediante la aplicación de la información corroboro cuales mantienen un manual de funciones y descripción de cargos en aplicación, y cuales no ejecutan esta herramienta de trabajo, de esta manera la información se puede utilizar como referencia para futuras investigaciones.

\section{Limitación de la investigación}

En la presente investigación no fue fácil acceder con rapidez a la información necesaria, por la limitación del tiempo del personal que debía proporcionarla; lo cual fue superado con la colaboración de los empleados de las organizaciones, ya que nos brindaron la información requerida para poder continuar con el análisis y propuesta de este proyecto. 
Otro motivo que se presentó en la investigación fue la cantidad de empresas a encuestar, ya que el $4.99 \%$ es la representación porcentual de las micro y pequeñas empresas concentradas en la provincia de El Oro equivalente a un total de 42.063 empresas donde se determinó que la muestra de la población a encuestar es de 381 empresas.

\section{Resultados}

Mediante la encuesta, se pudo conocer que actualmente el 68,8\% de las micro y pequeñas empresas de la provincia no cuentan con una manual de funciones, mientras que el 31,2\% restante si lo hacen. En este 31,2\% se encuentran aquellas empresas $(29,2 \%)$ que poseen un manual de funciones definido y lo aplican, asimismo, se encuentran aquellas empresas $(2,1 \%)$ que, a pesar de contar con un manual de funciones, este no es considerado para su aplicación.

Con respecto a las razones por las cuales ciertas empresas no cuentan actualmente con un manual de funciones, se pudo determinar que, la mayor parte de estas empresas $(51,9 \%)$ simplemente no consideran necesario aplicarlo, esto generalmente debido al reducido número de empleados con los que cuentan. Mientras que, el (25,9\%) afirma que actualmente se encuentran trabajando en la elaboración de un manual de funciones.

Del resto de empresas que no cuentan con un manual de funciones, estas supieron manifestar que anteriormente si poseían uno, pero no se lo aplicaba y otros manifestaron que simplemente desconocen la utilidad de los mismos. Por otra parte, del grupo de empresas que indicaron sí contar con un manual de empresas definido, la mayor parte de estas consideran que el uso de un manual de funciones es muy importante para el desempeñar de manera eficiente sus actividades. Uno de los puntos a resaltar dentro de la encuesta realizada es la incógnita que hace hincapié en la existencia de conflictos y sus causas en las operaciones administrativas, con la finalidad de relacionarlas con el uso del manual de funciones tal como se observa en la Figura 1.

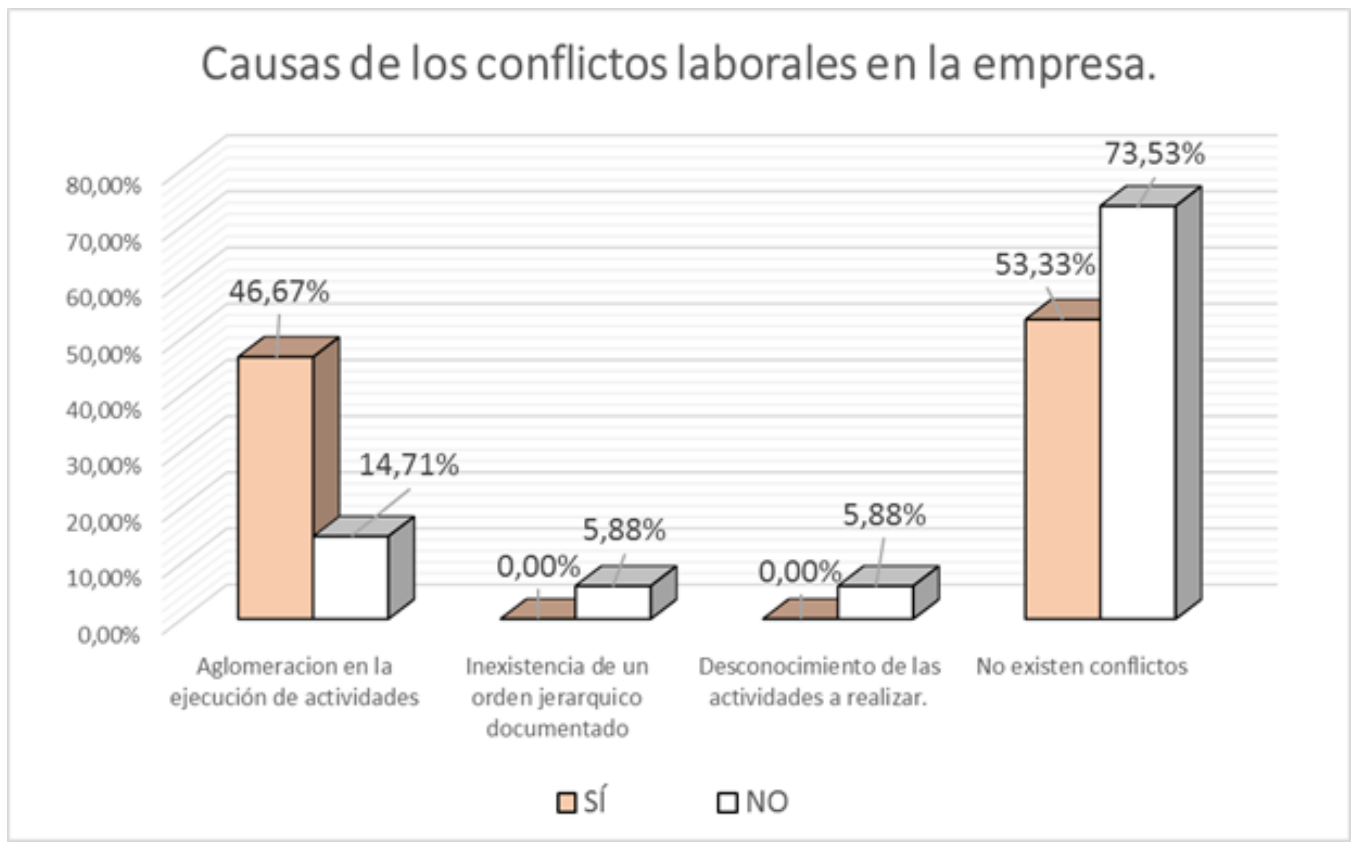


Figura 1: Causas de los conflictos laborales en la empresa. Fuente: Elaborado en base a los resultados de la encuesta.

Según los datos obtenidos el $(73,53 \%)$ de las empresas encuestadas que no tienen manual de funciones mencionan que no tienen conflictos, mientras que el $(53,33 \%)$ que si poseen un manual de funciones indican que presentan conflictos, el $(5,88 \%)$ que no utilizan un manual de funciones presentan desconocimiento de las actividades a realizar, mientras que las empresas que si poseen manual de funciones indican que conocen de sus actividades en totalidad, el $(5,88$ $\%$ ) de empresas encuestadas que no poseen manual de funciones mencionan que presentan un orden jerárquico documentado, mientras que los que sí poseen un manual de funciones resaltan que cuentan con un orden jerárquico documentado, finalmente el $(71.14 \%)$ las empresas que no poseen manual de funciones presentan aglomeración en la actividades a realizar.

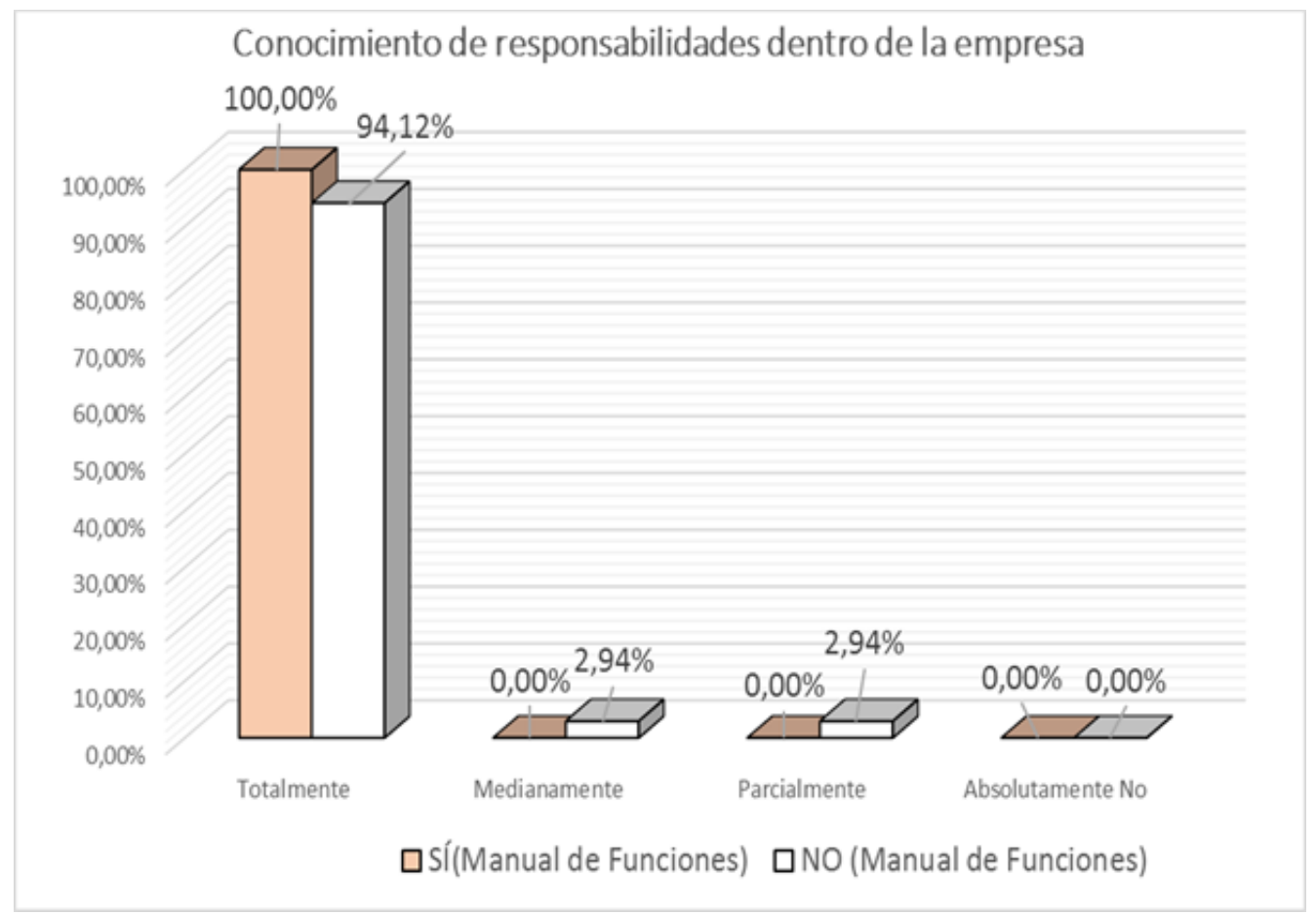

Figura 2: Conocimiento de responsabilidades dentro de la empresa. Fuente: en base a los resultados de la encuesta.

Asimismo, se pudo determinar que sin importar si la empresa cuenta o no con un manual de funciones, prácticamente todos los empleados conocen en su totalidad cuáles son sus responsabilidades dentro de la organización (Ver Figura 2). 


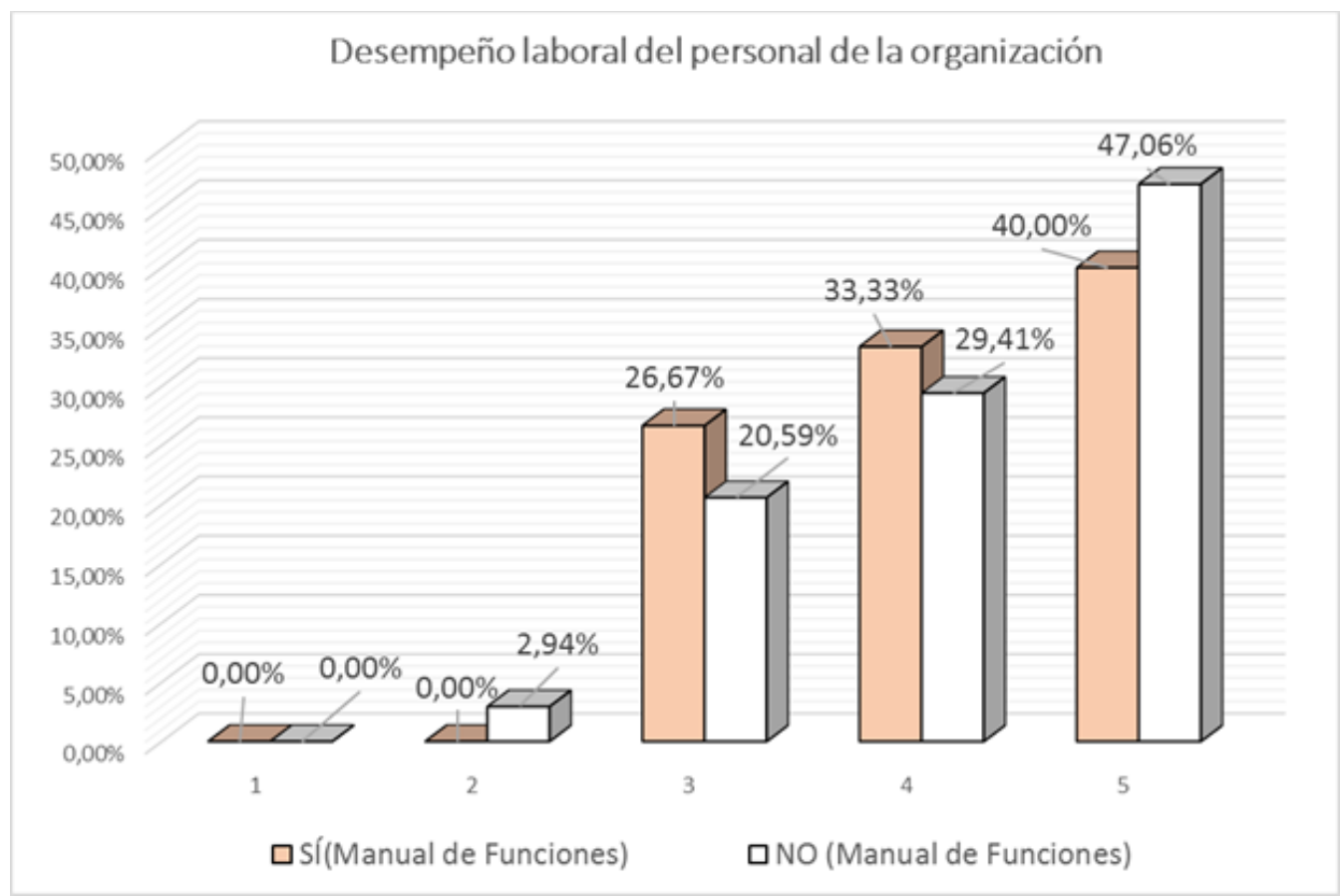

Figura 3: Desempeño laboral del personal de la organización. Fuente: en base a los resultados de la encuesta.

La siguiente incógnita fue aplicada con la finalidad de vincular el desempeño laboral y actividades diarias que realiza talento humano dentro de las empresas encuestadas, como se muestra en la

Figura 3, Los datos obtenidos muestran que la mayoría de las empresas mantienen un personal totalmente eficiente a pesar de contar o no con la aplicación de un manual de funciones, sin embargo, se pudo determinar que las empresas consideran de suma importancia su implementación en un futuro. 


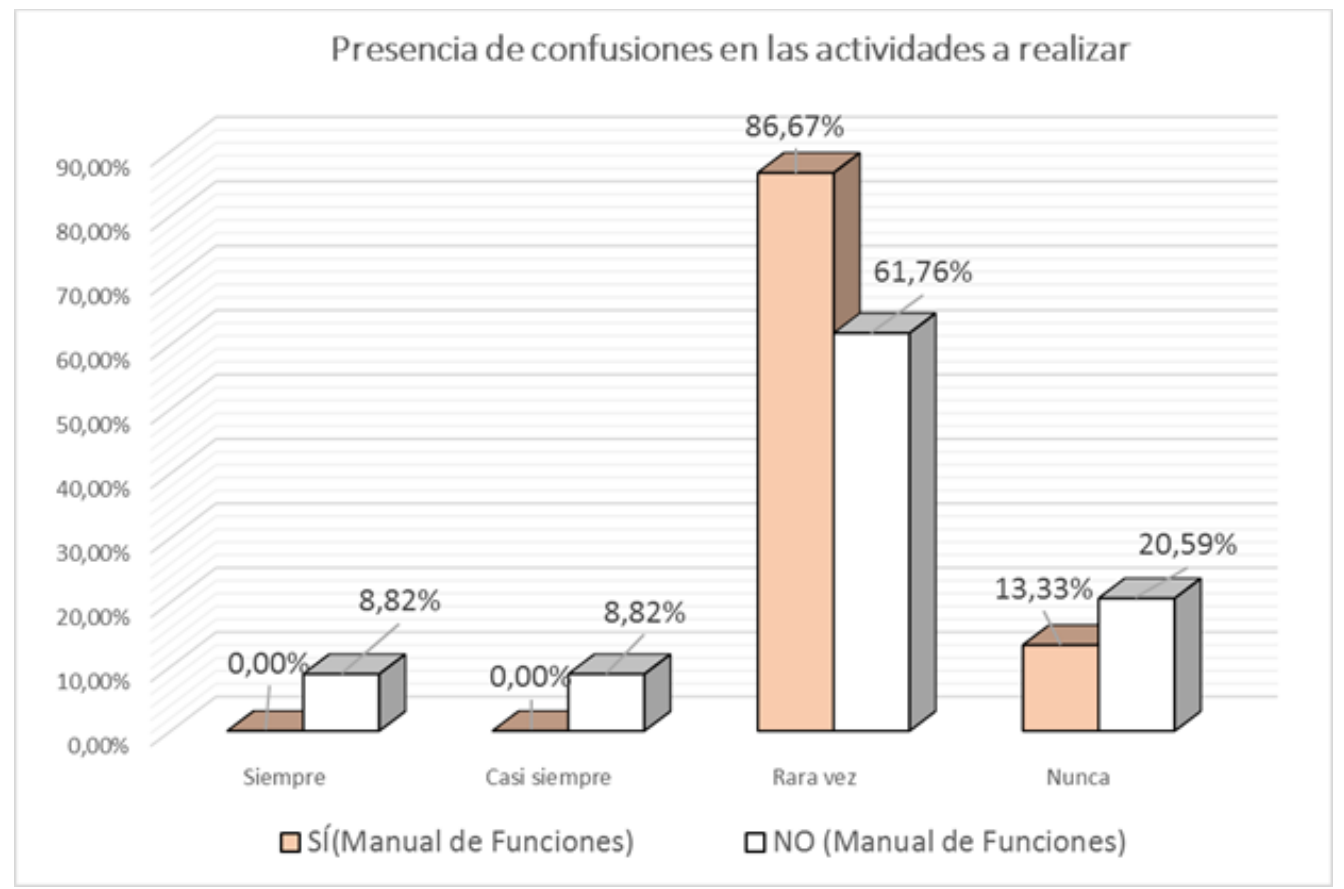

Figura 4: Presencia de confusiones en las actividades a realizar. Fuente: base a los resultados de la encuesta

A pesar de que los empleados conocen acerca de sus responsabilidades, aun así, se presentan confusiones en cuanto a sus actividades a realizar, siendo aquellas empresas que no poseen manual de funciones quienes presencian con mayor frecuencia estas ineficiencias. Cabe destacar que la diferencia es mínima, es decir, la existencia o no de un manual de funciones no influyen de gran manera en cuanto a estos aspectos (Ver Figura 4).

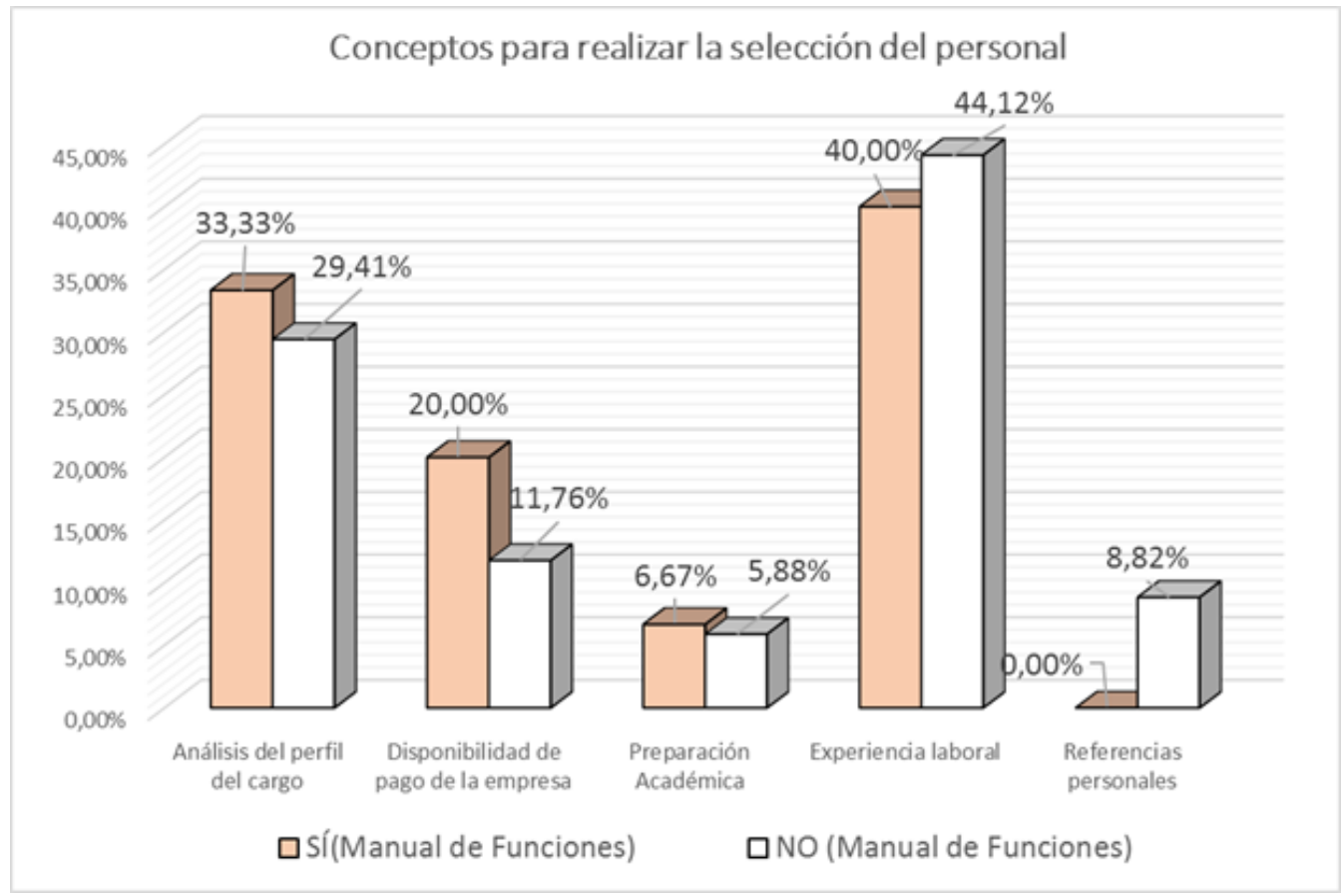

Figura 5: Conceptos para realizar la selección del personal. 


\section{Fuente: en base a los resultados de la encuesta}

En cuanto a los principales conceptos que las micro y pequeñas empresas toman en consideración al momento de efectuar la contratación de personal, estas toman en cuenta la experiencia laboral como requerimiento esencial (Ver Figura 5). Además, se determinó que una de las principales razones por la cual las empresas no aplican de un manual de funciones es el escaso conocimiento del mismo

\section{Discusión}

Luego de una deliberación grupal de los resultados expuestos detonan que efectivamente existe conflictos laborales internos en las micro y pequeñas empresas de El Oro en contextos como confusión al momento de ejecutar las actividades encomendadas, como también desconocimiento pleno o parcial de las responsabilidades de cada empleado, estos indicadores afectan directamente al desempeño laboral de cada organización.

Enfatizando los resultados obtenidos, se acepta la hipótesis planteada: La carencia de un manual de funciones da origen a desconciertos laborales, conflictos organizacionales, poca captación de responsabilidades de cargos, bajos estándares de formación e información al personal trabajador en micro y pequeñas empresas. Basándonos en la investigación documental para la elaboración del artículo científico contrastamos que es importante el manejo de conceptos básicos de las herramientas de gestión como es un manual de funciones, puesto que, dentro de las empresas encuestadas es notoria el desconocimiento pleno de ello, no obstante, existen empresas que conocen del mismo, mas no hacen el uso del mismo.

Con la recopilación de información de logra deducir tanto en la figura 1, como en la figura 4, que una empresa de menor dimensión como las microempresas pueden mantener un ambiente laboral ameno y debido a su reducido número de empleados mencionan que no lo consideran necesario, pero con la afirmación de que el mayor conflicto es la confusión de actividades y aglomeración de las mismas es vital mantener un manual de funciones documentado que respalde al empleado cuando no cuente en presencia del encargado. En cuanto las pequeñas empresas con un número de empleados más amplio, aplican un manual de funciones documentado, no obstante, reflejan que su mayor problemática se debe a la aglomeración de actividades, véase en Figura 1, debido a que no siempre se mantiene una correcta formalización del mismo.

En conclusión, la información recopilada releva que a pesar de mantener un personal eficiente e indistintamente si en la actualidad aplican o no un respectivo manual de funciones presenta problemáticas en sus actividades diarias, la asignación de cargos tiende a ser ambigua causante de que existan rara vez confusiones en la jornada laboral. Para complementar lo expuesto, se recomienda que para futuros estudios posteriores se aplique este mismo diseño, evaluando a estos mismos grupos de empresas, con la finalidad de comprobar si lo mencionado en el estudio está presto a mejoría del ambiente laboral en relación al rendimiento de los empleados tanto de micro y pequeñas empresas de El Oro

\section{Bibliografía}


Abreu, J. L. (2012). Hipótesis, Método \& Diseño de Investigación. Daena: International Journal of Good Conscience, 7(2), 187-197.

Arias Valencia, M. M. (2013). La triangulación metodológica: sus principios, alcances y limitaciones. Investigación y educación en enfermería, 23.

Bernal Domínguez, D., \& Herrera Caballero, J. M. (2014). Sobre la transferencia de modelos Organizacionales. Gestión y política Pública, 29. Obtenido de http://www.redalyc.org/articulo.oa?id=13331693002

Campos y Covarrubias, G., \& Lule Martínez, N. E. (2013). La Observación, Un Método Para El Estudio De La Realidad. Xihmai, 1.

Castañeda, D. I., \& Fernández Ríos, M. (2007). Validación de una escala de niveles y condiciones de aprendizaje Organizacional. Universidad Psychologica, 11. Obtenido de http://www.redalyc.org/articulo.oa?id=64760204

Castañeda, D. I. (2015). Condiciones para el aprendizaje organizacional. Estudios Gerenciales, 7. Obtenido de http://www.redalyc.org/articulo.oa?id=21233043007

Cauas, D. (2015). Definición de las variables, enfoque y tipo. Biblioteca electrónica de la Universidad Nacional, 5. Obtenido de http://datateca. unad. edu. Co/contenidos/210115/Documento_reconociemiento_Unidad_N o_2. Pdf\#

Comunidad Andina De Naciones. (2009). Resolución 1260.

Delgado, F. (2014). SMS Ecuador. Obtenido de SMS Auditores del Ecuador Cia.Ltda: http://www.smsecuador.ec/manual-de-funciones/

El Telégrafo. (Dic de 7 de 2016). Obtenido de http://www.eltelegrafo.com.ec/noticias/economia/8/la-junta-monetaria-elevo-el-encajepara-grandes-bancos

Ibáñez, C. L., \& Egoscozábal, A. M. (2008). Metodologías de la investigación en las ciencias sociales: Fases, fuentes y selección de técnicas. Revista Escuela de Administración de Negocios, 5-18.

INEC. (2015). Directorio de empresas y establecimientos. Ecuador.

León, L. (septiembre de 2013). Implementación de un manual de funciones en el Área Administrativa de la compañía Torres \& Asociados S. A. Milagro, Ecuador: Universidad Estatal de Milagro. Obtenido de http://repositorio.unemi.edu.ec/handle/123456789/610

Loyo de Falcón, R. E. (2015). La cultura organizacional desde la perspectiva del docente Unefa. Omnia, 19. Obtenido de http://www.redalyc.org/articulo.oa?id=73743366004 
Magill J, M. (2004). Micro Empresas y Micro Finanzas. Usaid, 192.

Martínez, L. (2007). La observación y el diario de campo en la definición de un tema de investigación. Revista perfiles libertadores, 73-80.

Paz, G. M. (2014). Metodología de la Investigación. México: Grupo Editorial Patria.

Pita Fernández, S., \& Pértegas Díaz, S. (2002). Investigación cuantitativa y cualitativa. Cad Aten Primaria, 76-78.

Sotomayor Cún, A. L. (2016). Diseño de un manual de funciones para Credi Comercial Yumbai de la ciudad de Arenillas en periodo 2014 (Trabajo de Titulación) Utmach. Unidad Académica de Ciencias Empresariales, 30.

Utreras, J. M. (2015). La investigación científica de la comunicación en América latina. Chaqui. 